\title{
Influência do ciclo estral sobre a sensibilidade da resposta cronotrópica à norepinefrina em ratas submetidas a estresse agudo
}

\author{
Ana Paula Tanno, Fábio José Bianchi, Fernanda Klein Marcondes* \\ Departamento de Ciências Fisiológicas, Faculdade de Odontologia de Piracicaba, FOP/UNICAMP, Piracicaba - SP
}

*Correspondência:

F. K. Marcondes.

Departamento de Ciências

Fisiológicas.

Faculdade de Odontologia de

Piracicaba - FOP/UNICAMP.

Av. Limeira, 901, 13414-903

Piracicaba/SP.

E-mail: fklein@fop.unicamp.br
O estresse pode alterar a sensibilidade da resposta cronotrópica às catecolaminas em vários tecidos. $O$ objetivo deste estudo foi avaliar a sensibilidade à norepinefrina (NE) em átrios direitos de ratas submetidas ao estresse agudo por natação nas fases de estro e proestro. Ratas Wistar em estro ou proestro foram submetidas a uma sessão de 50 min de natação, após a qual foram anestesiadas e sacrificadas. Os átrios direitos destes animais e de ratas controle foram isolados para obtenção de curvas concentração-efeito à NE antes e após o bloqueio dos sistemas de metabolização das catecolaminas (fenoxibenzamina + estradiol). Os dados foram analisados por ANOVA ou teste $t$ de Student. Não houve diferenças de sensibilidade à NE entre as fases de estro e proestro nos tecidos isolados de animais controle $(p>0,05)$. No proestro, a natação induziu supersensibilidade à $N E(p<0,05)$ sem alteração na fase de estro. No proestro, o desvio observado após o bloqueio dos sistemas de metabolização foi menor no tecido de animais estressados em relação ao tecido isolado de ratas controle $(1,7 x$ 2,4; $p<0,05)$. A supersensibilidade à NE induzida por uma sessão de natação aplicada no proestro pode ser parcialmente explicada por uma inibição nos processos de metabolização das catecolaminas.

\author{
Unitermos: \\ - Estresse \\ - Natação \\ - Norepinefrina \\ - Ratas \\ - Supersensibilidade \\ - Estro \\ - Proestro
}

\section{INTRODUÇÃO}

O estresse é uma experiência de vida diária e todos os organismos sofrem alterações em seu meio externo e interno para enfrentar os agentes estressores, os quais ativam um amplo espectro de sistemas neuronais e hormonais, induzindo alterações fisiológicas e comportamentais para promover a adaptação à nova situação (Flugge, 2000; Jasmin, Bois, 2000). Frente a uma ameaça física, esta é uma resposta eficiente para garantir a sobrevivência, bem como aumentar a capacidade do organismo para a fuga (Lundberg, 2000). Os estressores que participam normalmente do co- tidiano dos seres humanos podem alterar o funcionamento do organismo e contribuir para doenças degenerativas e envelhecimento (McCann et al., 2000).

Embora a reação de estresse possa resultar em doenças, é ela que torna possível a sobrevivência e a adaptação dos seres vivos frente aos inúmeros estímulos ambientais a que estão constantemente expostos (Fraser et al., 1975; Chrousos, Gold, 1992).

Os principais mediadores da resposta ao estresse são as catecolaminas (norepinefrina (NE) e epinefrina), liberadas pelo sistema nervoso simpático e pela medula da adrenal, e os glicocorticóides liberados pelo córtex da adrenal. As 
funções cardiovasculares e neuroendócrinas ativadas pelas catecolaminas mobilizam energia para os músculos, coração, cérebro e, ao mesmo tempo, reduzem o fluxo sanguíneo para outros órgãos, como o sistema gastrintestinal. As catecolaminas e os glicocorticóides exercem eventos celulares que promovem mudanças adaptativas em células e tecidos, com a função de manter a homeostasia e promover a sobrevivência do organismo (McEwen, 2000).

As catecolaminas exercem suas ações por interação com os receptores de membrana, denominados adrenoceptores. Quando as catecolaminas ocupam esses receptores ocorre a formação de segundos mensageiros intracelulares, que desencadeiam a resposta fisiológica. A resposta dos tecidos às catecolaminas é resultante não só de sua interação com receptores adrenérgicos, e dos eventos intracelulares que se seguem a essa interação, mas também da atividade dos sistemas de metabolização das catecolaminas (Brodde, Michel, 1999; Kable et al., 2000).

As respostas desencadeadas pelo organismo frente a agentes estressores podem produzir alterações de sensibilidade adrenérgica no tecido atrial, como foi observado em ratos submetidos a estresse por frio (Harri et al., 1974; Callia, De Moraes, 1984), choques (Bassani, De Moraes, 1987; Spadari-Bratfisch et al., 1999), imobilização (Capaz, De Moraes, 1988) ou natação (Spadari et al., 1988; Marcondes, 1995).

Entretanto, a exposição ao mesmo agente estressor induz alterações diferentes, dependendo da qualidade do estímulo estressor e do sexo do animal. E, em ratas, tais alterações também dependem da fase do ciclo estral (Marcondes et al., 1996). Nosso objetivo neste trabalho foi avaliar a sensibilidade à norepinefrina (NE) em átrios direitos de ratas submetidas ao estresse agudo por natação nas fases de estro e proestro.

\section{MATERIAL E MÉTODOS}

Foram utilizadas ratas Wistar com 3,5-4,5 meses de idade. Os animais foram mantidos no Biotério da Faculdade de Odontologia de Piracicaba, alojados em gaiolas coletivas, com 5 animais, em sala climatizada $\left(22 \pm 2{ }^{\circ} \mathrm{C}\right)$, e com ciclo claro/escuro de 12/12 h (luzes acendendo às 6:00 h). Receberam, durante todo o período, água e ração para ratos à vontade. Todos os procedimentos utilizados neste estudo foram aprovados pelo Comitê de Ética na Experimentação Animal do Instituto de Biologia/ UNICAMP (CEEA - protocolo $\mathrm{n}^{\circ}$ 13-1).

A determinação das fases do ciclo estral foi feita diariamente entre 7:30 e 9:00 h, por observação do lavado vaginal durante, no mínimo, dois ciclos consecutivos (Marcondes et al., 2002).
Ratas em estro ou proestro foram submetidas, individualmente, a uma sessão de 50 min de natação em um tanque de acrílico medindo $50 \mathrm{~cm}$ x $50 \mathrm{~cm} \times 50 \mathrm{~cm}$, contendo água a $30 \pm 1{ }^{\circ} \mathrm{C}$, à profundidade de $38 \mathrm{~cm}$, suficiente para evitar que os animais encostassem a cauda no fundo do mesmo (Bianchi et al., 2001).

Imediatamente após as sessões de natação os animais foram anestesiados por inalação de halotano a $2 \% \mathrm{e}$ sacrificados por secção dos vasos cervicais (Bianchi, Tanno, Marcondes, 2001). O átrio direito foi preparado para registro isométrico das contrações espontâneas em câmara para órgãos isolados, contendo solução de KrebsHenseleit a $36,5 \pm 0,1{ }^{\circ} \mathrm{C}$, borbulhada continuamente com $95 \%$ de $\mathrm{O}_{2}$ e $5 \%$ de $\mathrm{CO}_{2}$ (Marcondes et al., 1996).

Após o período de estabilização (45-60 min) foram obtidas curvas concentração-efeito à NE pelo método cumulativo, com incrementos sucessivos da concentração molar do agonista de 0,5 unidade logarítmica (Van Rossum, 1963). A resposta máxima foi determinada quando três concentrações sucessivas e crescentes de NE não alteraram a resposta obtida com a concentração imediatamente anterior.

Ao final da primeira curva concentração-efeito à NE, o tecido foi lavado com solução de Krebs-Henseleit até que a freqüência retornasse ao nível obtido antes da realização da curva. Em seguida, para avaliação da atividade dos sistemas de metabolização das catecolaminas, o tecido foi pré-tratado com $10 \mu \mathrm{M}$ de fenoxibenzamina para bloqueio do sistema de recaptação neuronal e dos adrenoceptores alfa (Cubeddu et al., 1974; Eisenfeld et al., 1967; Zanesco et al., 1997). Após 30 min, o tecido foi lavado e a solução de incubação trocada a cada dez minutos durante uma hora, para remover o excesso de fenoxibenzamina. Em seguida, adicionou-se estradiol $(5 \mu \mathrm{M})$, bloqueador do sistema de captação extraneuronal (Salt, 1972), à solução de incubação, na presença do qual nova curva concentração-efeito à NE foi obtida. A sensibilidade do átrio direito foi avaliada pela determinação do valor $\mathrm{pD}_{2}$, que corresponde ao logaritmo negativo da concentração molar do agonista que determina $50 \%$ do efeito máximo, em experimentos individuais, utilizando o programa GraphPAD software San Diego, CA, USA (Moura, Marcondes, 2001).

Para a solução de Krebs-Henseleit foram utilizados sais de padrão analítico (Merck) e água desionizada. A NE (Sigma Chemical Co) foi diluída em solução de ácido ascórbico a $2 \%$ e armazenada a $-20{ }^{\circ} \mathrm{C}$, por uma semana. As diluições foram feitas em solução de Krebs-Henseleit imediatamente antes do uso. A fenoxibenzamina (RBI) e o estradiol (Merck) foram diluídos em etanol (Merck) e armazenados a $-20{ }^{\circ} \mathrm{C}$ durante um mês. 
Para avaliação do efeito do estresse e do ciclo estral foi realizada Análise de Variância bifatorial, com os fatores fase (proestro e estro) e tratamento (controle e estresse), seguida do teste de Tukey para comparações múltiplas de médias. Para a comparação dos dados referentes às curvas concentração-efeito obtidas no mesmo tecido foi utilizado teste $t$ de Student para amostras pareadas. Valores de $p$ menores do que 0,05 foram indicativos de significância estatística.

\section{RESULTADOS}

Para a análise da freqüência inicial foram utilizados os dados obtidos após a estabilização do tecido, imediatamente antes do início da primeira curva concentraçãoefeito. Os dados estão apresentados na Tabela I. Não foram observadas diferenças entre as fases de estro e proestro nos valores de freqüência inicial e resposta má-

TABELA I - Valores de freqüência inicial (FI) e resposta máxima (RM) à NE de átrios direitos isolados de ratas em estro e proestro controles ou submetidas a estresse por 50 minutos de natação

\begin{tabular}{lllcc}
\hline \multicolumn{2}{c}{ Grupos } & $\mathrm{N}^{\mathrm{a}}$ & $\begin{array}{c}\mathrm{FI}^{\mathrm{b}} \\
\text { bat/min }\end{array}$ & $\begin{array}{c}\mathrm{RM}^{\mathrm{b}} \\
\text { bat/min }\end{array}$ \\
\hline Estro & controle & 5 & $268 \pm 08$ & $180 \pm 06$ \\
\multirow{4}{*}{ Proestro } & Natação & 5 & $283 \pm 03$ & $175 \pm 15$ \\
& controle & 5 & $260 \pm 14$ & $197 \pm 14$ \\
& Natação & 5 & $261 \pm 06$ & $175 \pm 10$ \\
\hline
\end{tabular}

${ }^{a}$ Número de experimentos. ${ }^{b}$ Valores médios (acompanhados dos respectivos erros-padrão das médias). xima à $\mathrm{NE}$ em átrios direitos isolados de ratas controles (Tabela I, p>0,05). Não houve diferença de freqüência inicial e de resposta máxima entre os animais dos grupos controle e os respectivos grupos submetidos à natação (Tabela I; $\mathrm{p}>0,05$ ).

$\mathrm{Na}$ Tabela II estão apresentados os valores $\mathrm{pD}_{2}$ de $\mathrm{NE}$ em átrios direitos isolados de ratas submetidas a uma sessão de 50 minutos de natação. Não houve diferença entre as fases de estro e proestro nos valores de $\mathrm{pD}_{2}$ de $\mathrm{NE}$ em átrios direitos isolados de ratas controle (Tabela II curva $1 ; p>0,05)$.

O estresse por sessão única de natação não induziu alteração de sensibilidade à $\mathrm{NE}$ em átrios direitos isolados de ratas em estro (Tabela II-curva 1, Figura 1, p>0,05). Entretanto, houve supersensibilidade à catecolamina em átrios direitos isolados de ratas em proestro submetidas a 50 minutos de natação, em relação ao respectivo grupo controle, com um deslocamento significativo à esquerda de 2,4 vezes na curva concentração-efeito à NE (Tabela IIcurva 1, Figura $1 ; p<0,05$ ).

Para avaliarmos a participação dos processos de recaptação neuronal e captação extraneuronal nas alterações que foram observadas, foram realizadas curvas concentração-efeito à NE, antes e após tratamento farmacológico in vitro do tecido atrial para inibição dos processos de metabolização. Na Tabela II estão apresentados os valores $\mathrm{pD}_{2}$ da NE obtidos na $2^{\mathrm{a}}$ curva concentração-efeito, realizada no mesmo tecido, na presença de bloqueadores dos sistemas de metabolização de catecolaminas. Independentemente da fase do ciclo estral e do tratamento (controle ou estresse), ocorreu um desvio significativo à esquerda da curva concentração-efeito à NE (Tabela II; $\mathrm{p}<0,05)$.

TABELA II - Valores $\mathrm{pD}_{2}$ da NE de átrios direitos isolados de ratas em estro e proestro controles e submetidos a estresse por 50 min de natação antes e após bloqueio dos sistemas de metabolização

\begin{tabular}{llcccc}
\hline \multicolumn{2}{c}{ Grupo } & $\mathrm{N}^{\mathrm{a}}$ & $\mathrm{pD}_{2}{ }^{\mathrm{b}}$ curva $1^{\mathrm{c}}$ & $\mathrm{pD}_{2}{ }^{\mathrm{b}}$ curva 2d & Desvio $^{\mathrm{e}}$ \\
\hline \multirow{2}{*}{ Estro } & controle & 5 & $7,20 \pm 0,15$ & $7,63 \pm 0,08 \#$ & 2,69 \\
\multirow{3}{*}{ Proestro } & natação & 5 & $7,27 \pm 0,05$ & $7,69 \pm 0,04 \#$ & 2,75 \\
& controle & 5 & $7,14 \pm 0,03$ & $7,52 \pm 0,01 \#$ & 2,42 \\
& natação & 5 & $7,55 \pm 0,04^{*}$ & $7,77 \pm 0,03 \# *$ & $1,71^{*}$ \\
\hline
\end{tabular}

${ }^{a}$ Número de experimentos. ${ }^{b}$ Valores médios (acompanhados dos repectivos erros padrão das médias) dos logaritmos negativos da concentração molar de NE que determinou $50 \%$ da resposta máxima nos tecidos isolados . ${ }^{\circ}$ Curva realizada na ausência de inibidores de metabolização das catecolaminas. ${ }^{\mathrm{d} C}$ urva realizada na presença de inibidores dos processos de metabolização neuronal e extraneuronal. ${ }^{~}$ Valores médios dos desvios entre a curva concentração-efeito à NE obtida na presença e na ausência de inibidores de metabolização das catecolaminas. *Estatisticamente diferente do respectivo grupo controle $(\mathrm{p}<0,05$; ANOVA Bifatorial). \# Estatisticamente diferente da respectiva curva realizada no mesmo tecido antes do bloqueio dos sistemas de metabolização ( $p<0,05$; teste t pareado). 


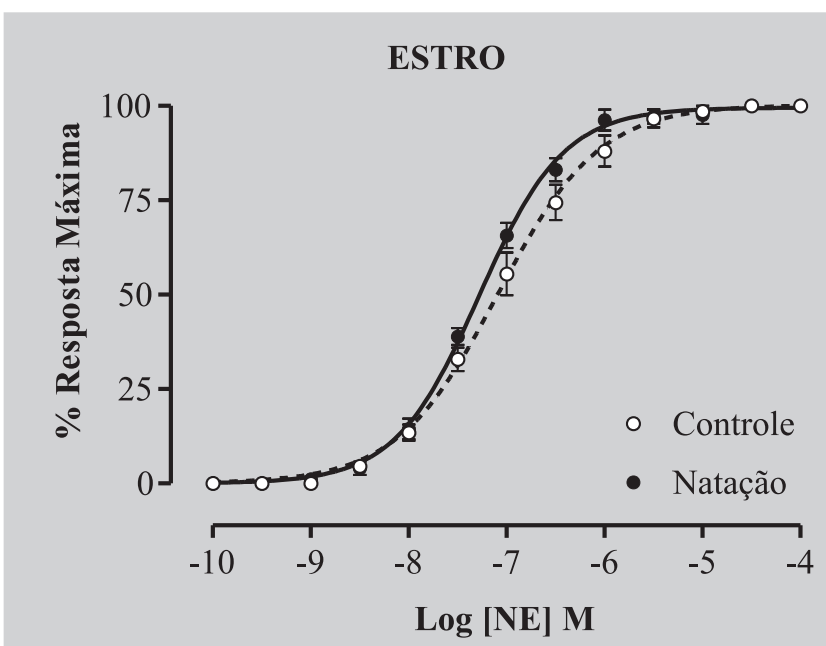

PROESTRO

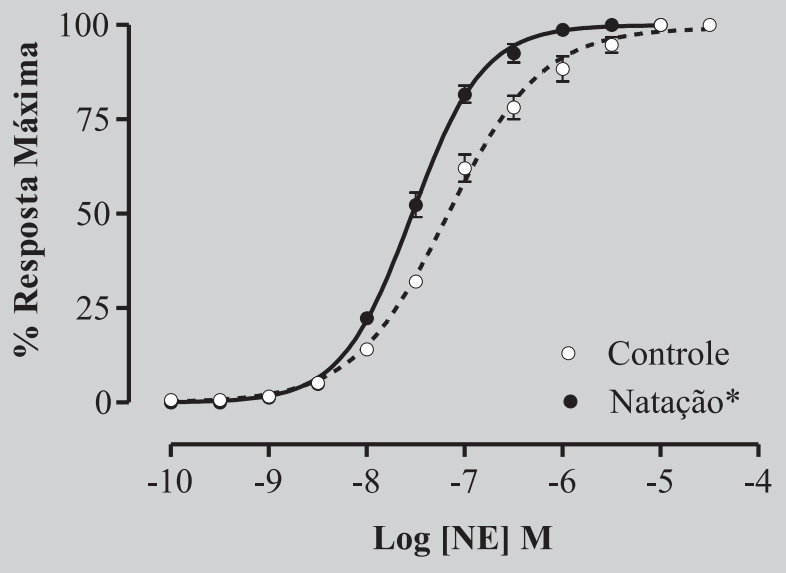

FIGURA 1 - Curvas concentração-efeito à NE em átrios direitos de ratas controle ou submetidas à natação durante o estro e o proestro. *Estatisticamente diferente do respectivo grupo controle ( $\mathrm{p}<0,05$; ANOVA + Tukey).

Durante o estro, a magnitude dos desvios observados foi semelhante em animais controles e estressados (Tabela II; $p>0,05$ ). Entretanto, durante o proestro, o desvio causado pelo bloqueio dos sistemas de metabolização das catecolaminas em átrios direitos isolados de ratas submetidas à natação foi menor em relação aquele observado nos tecidos isolados de animais controles (Tabela II; $\mathrm{p}<0,05)$. Nas curvas concentração-efeito à $\mathrm{NE}$ obtidas na presença de bloqueadores dos sistemas de metabolização das catecolaminas também foi observada supersensibilidade à catecolamina em átrios direitos isolados de ratas em proestro submetidas à natação, em relação ao grupo controle (Tabela II; $\mathrm{p}<0,05$ ).

\section{DISCUSSÃO}

A natação, além do componente físico, apresenta um forte componente emocional, relacionado à novidade que este estímulo representa para o rato de laboratório, à impossibilidade de fuga e à iminência de morte (ÖstmanSmith, 1979; Cox et al., 1985; Garcia-Marquez, Armario, 1987). Ao longo da sessão de natação, o animal passa a alternar momentos de nado ativo com momentos de imobilidade, diminuindo o esforço físico, desenvolvendo, assim, uma resposta adaptativa (Bruner, Vargas, 1994).

Os resultados aqui apresentados mostram que o protocolo de estresse por sessão única de natação induziu supersensibilidade aos efeitos cronotrópicos da NE em átrios direitos de ratas na fase de proestro, sem alterações na fase de estro.

As respostas desencadeadas pelo organismo, frente a agentes estressores, podem produzir alterações de sensibilidade adrenérgica no tecido atrial, como foi observado em ratos submetidos a estresse por frio (Harri et al., 1974; Callia, De Moraes, 1984), choques (Bassani, De Moraes, 1987; Spadari-Bratfisch et al., 1999), imobilização (Capaz, De Moraes, 1988) ou natação (Spadari et al., 1988; Marcondes, 1995).

Em fêmeas, o estresse repetido por choque nas patas e natação induziu alterações de sensibilidade aos efeitos cronotrópicos da epinefrina e da NE quando o animal foi sacrificado no diestro, sem alterações no estro. Os autores concluíram que as alterações de sensibilidade do tecido atrial são dependentes das fases do ciclo estral. (Marcondes, 1995; Marcondes et al., 1996; Vanderlei et al., 1996).

Neste contexto, nossos resultados confirmam a influência do ciclo reprodutivo e do modelo de estresse nos mecanismos adaptativos desencadeados sobre o sistema cardiovascular.

As alterações de sensibilidade adrenérgica podem ocorrer por meio de vários mecanismos: alterações da atividade dos processos de recaptação neuronal e captação extraneuronal (Bassani, De Moraes, 1987; Spadari et al., 1988), modificações na estrutura (Callia, De Moraes, 1984; Spadari, De Moraes, 1988) ou na densidade dos adrenoceptors $\alpha$ e $\beta$ (Mano et al., 1979; Flügge, 2000), ou indução de heterogeneidade da população de adrenoceptores $\beta$, que medeiam as respostas às catecolaminas (Callia, De Moraes, 1984; Bassani, de Moraes, 1988).

No presente estudo o bloqueio dos sistemas de metabolização das catecolaminas provocou um desvio significativo à esquerda, independente da fase do ciclo estral, na curva concentração-efeito à NE. Ao comparar- 
mos curvas concentração-efeito na ausência e na presença de inibidores dos mecanismos de metabolização, espera-se que a inibição de tais processos determine desvio significativo à esquerda na curva na presença dos inibidores (Kenakin, 1993). Se isto não ocorrer, o sistema analisado provavelmente encontra-se inibido por outro fator, que não o tratamento in vitro (Rodrigues et al., 1995; Zanesco et al., 1997).

O desvio observado no estro se deve ao efeito do bloqueio in vitro dos sistemas de metabolização. Ao compararmos curvas concentração-efeito na ausência e na presença de inibidores dos mecanismos de metabolização, evidenciamos que a atividade destes sistemas não difere entre ratas controles e estressadas. Entretanto, na fase de proestro, observou-se desvio menor nos animais submetidos à natação, quando comparados aos respectivos controles (1,71 x 2,42 vezes), sugerindo uma alteração, nesta fase do ciclo estral, dos processos de metabolização das catecolaminas.

Portanto, a supersensibilidade da resposta cronotrópica à NE induzida pelo estresse na fase de proestro parece envolver uma alteração nos processos de recaptação neuronal e captação extraneuronal das catecolaminas. Porém, outros mecanismos também parecem estar envolvidos, tendo em vista que o bloqueio dos mecanismos de metabolização não cancelou a supersensibilidade à NE induzida pelo estresse.

Embora a NE seja principalmente metabolizada pelo sistema de recaptação neuronal, em situações de estresse seus níveis plasmáticos apresentam-se elevados e o sistema de captação extraneuronal passa a ser importante na finalização de seus efeitos nos tecidos alvo (Vanderlei et al., 1996).

A eficiência dos sistemas de captação extraneuronal e de recaptação neuronal pode ser alterada pela ação de diversas substâncias, tais como a corticosterona, o estradiol e a progesterona (Collins et al., 1970; Iversen, Salt, 1970; Salt, 1972; Kopin, 1985).

Durante o ciclo estral ocorrem variações dos níveis plasmáticos de corticosterona e de esteróides sexuais. Os menores níveis de estradiol são observados durante o estro, ocorrendo aumento no metaestro para atingir o pico no proestro (Smith et al., 1975; Freeman, 1988). Com relação à progesterona, ocorre um pico durante o metaestro e outro durante o proestro, sendo os menores níveis observados durante o diestro (Smith et al., 1975; Freeman, 1988). As concentrações plasmáticas de corticosterona são elevadas durante o proestro, quando comparadas com outras fases do ciclo (Rodrigues et al., 1995).
Os níveis plasmáticos de corticosterona e de esteróides sexuais também podem ser alterados por situações de estresse (Marcondes, 1998; Shors et al., 1999). Alterações nas concentrações de corticosterona e/ou progesterona e/ou estradiol e na proporção entre estradiol e progesterona, induzidas pelo estresse, poderiam estar relacionadas com a alteração de sensibilidade à NE, que foram observadas na fase de proestro. Esta hipótese está sendo estudada em nosso laboratório.

\section{ABSTRACT}

\section{Influence of estrous cycle on the sensitivity of cronotropic answer to norepinephrine in rats submitted to acute stress}

Stress may change the response to catecholamines in many tissues. The aim of this study was to investigate the influence of the estrous cycle on the sensitivity to norepinephrine in right atria from female rats submitted to a single swimming session. Wistar female rats were submitted to one swimming session at estrus or proestrus. Immediately after the stress session, the animal was sacrificed and its right atria set up for isometric recording of spontaneous beating. Concentration-effect curves to norepinephrine were obtained before and after inhibition of uptake (phenoxibenzamine) and uptake (estradiol). Swimming stress did not change the sensitivity to noradrenaline in right atria from rats at estrus. However, at proestrus swimming induced supersensitivity to norepinephrine ( $\mathrm{D}_{2}$ control: $7.14 \pm 0.03 \mathrm{vs} . \mathrm{p} \mathrm{D}_{2}$ swimming: $7.55 \pm 0.04 ; p<0.05)$. Moreover at proestrus, the inhibition of the uptake systems induced a lower shift to the left in the concentration-effect curves to norepinephrine compared to the estrus. Changes on the uptake systems seem to be involved in the stress-induced supersensitivity to norepinephrine during proestrus.

UNITERMS: Swimming stress. Norepinephrine. Female rats. Supersensitivity. Estrus. Proestrus

\section{AGRADECIMENTOS}

Este estudo foi financiado por auxílios-pesquisa concedidos a FKM pela Fundação de Apoio a Pesquisa do Estado de São Paulo (FAPESP 99/00793-1) e Fundo de Apoio ao Ensino e Pesquisa (FAEP/UNICAMP 0027/00). APT e FJB receberam bolsas FAPESP de mestrado (99/11442-5) e iniciação científica (99/02358-0), respectivamente. 


\section{REFERÊNCIAS BIBLIOGRÁFICAS}

BASSANI, R. A., DE MORAES, S. Effects of repeated footshock stress on the chronotropic responsiveness of the isolated pacemaker of the rat: role of $\beta_{2}$ adrenoceptors. J. Pharmacol. Exp. Ther, Baltimore, v. 246, n. 1, p. 316-321, 1988.

BASSANI, R. A., DE MORAES, S. Subsensitivity to beta adrenoceptor agonists in right atria isolated from footshock stressed rats. Gen. Pharmacol., Oxford, v. 18, n. 5, p. 473-477, 1987.

BIANCHI, F. J., TANNO, A. P., MARCONDES, F. K. Relação entre nível de estresse e supersensibilidade à norepinefrina em ratas no poestro. Rev. Bras. Ciênc. Farm., v. 37, n. 3, p. 391-398, 2001.

BRODDE, O. E. , MICHEL, M. C. Adrenergic and Muscarinic Receptors in the Human Heart. Pharmacol. Rev., Baltimore, v. 51, n. 4, p. 651-689, 1999.

BRUNER, C. A. , VARGAS, I. The activity of rats in a swimming situation as a function of water temperature. Physiol. Behav., New York, v. 55, n. 1, p. 21-28, 1994.

CALLIA, M. L. , DE MORAES, S. Heterogeneity of beta adrenoceptors in right atria isolated from cold-exposed rats. J. Pharmacol. Exp. Ther., Baltimore, v. 230, n. 2, p. 450-454, 1984.

CAPAZ, F. R. , DE MORAES, S. Reduction by acute restraint stress of norepinephrine sensitivity in the isolated rat pacemaker. Eur. J. Pharmacol., Amsterdam, v. 147, n. 2, p. 295-298, 1988.

CHROUSOS, G. P. , GOLD, P. W. The concepts of stress and stress system disorders. Overview of physical and behavioral homeostasis. J. Am. Med. Ass., Chicago, v. 267, n. 9, p. 1244-1252, 1992.

COLLINS, G. G. S., PRYSE-DAVIES, J., SANDLER, M., SOUTHGATE, J. Effect of tretreatment with oestradiol, progesterone and DOPA on monoamino oxidase activity in the rat. Nature, London, v. 226, n. 246, p. 642-643, 1970.

COX, R. H., HUBBARD, J. W., LAWLER, J. E., SANDERS, B. J. , MITCHELL, V. P. Cardiovascular a sympathoadrenal responses to stress in swim-trained rats. J. Appl. Physiol. Bethesda, v. 58, n. 4, p. 1207-1214, 1985.
CUBEDDU, L., LANGER, S.Z. , WEINER, N. The relationships between alpha receptor block, inhibition of norepinephrine uptake and the release and metabolism of ${ }^{3} \mathrm{H}$-norepinephrine. J. Pharmacol. Exp. Ther., Baltimore, v. 188, n.2, p. 368-385, 1974.

EISENFELD, A.J., AXELROD, J., KRAFOFF, L. Inhibition of the extraneuronal accumulation and metabolism of norepinephrine by adrenergic blocking agents. $J$. Pharmacol. Exp. Ther., Baltimore, v. 156, p. 107-113, 1967.

FLÜGGE, G. Regulation of monoamine receptors in the brain: dynamic changes during stress. Int. Rev. Citol., New York, v. 195, p. 145-213, 2000.

FRASER, D., RITCHIE, J. S. D. , FRASER, A. F. The term "stress" in a veterinary context. Br. Vet. J., London, v. 131, n. 6 , p. 653-62, 1975.

FREEMAN, M. E. The ovarian cycle of the rat. In: KNOBIL, E. et al. The physiology of reproduction. New York: Raven Press. LTD, 1988. p. 1893-1928.

GARCIA-MARQUEZ, C. G. , ARMARIO, A. Chronic stress depresses exploratory activity and behavioral performance in the forced swimming test without altering ACTH response to a novel acute stressor. Physiol. Behav., New York, v. 40, p. 33-38, 1987.

HARRI, M. N. E., MELENDER, L. , TIRRI, R. Changed chronotropic sensitivity to sympathomimetic amines in isolated atria from rats following cold acclimation. Experientia., Basel, v. 30, n. 9, p. 1041-1043, 1974.

IVERSEN, L. L., SALT, P. J. Inhibition of catecholamine uptake $_{2}$ by steroids in the isolated rat heart. $B r . J$. Pharmacol., Basingstoke, v. 40, n. 3, p. 528-530, 1970.

JASMIN, G. , BOIS, P. Hans Selye. In: FINK, G., ed. Encyclopedia of Stress. New York: Academic Press, 2000. v. 3, p. 417-418

KABLE, J. H., MURRIN, C. , BYLUND, D. B. In Vivo Gene Modification Elucidates Subtype-Specific Functions of $\mathrm{a}_{2}$-Adrenergic Receptors. J. Pharmacol. Exp. Ther., Baltimore, v. 293, n. 1, p. 1-7, 2000.

KENAKIN, T. P. Pharmacologic analysis of drug-receptor interaction. 2. ed. New York: Raven Press, 1993. 483 p. 
KOPIN, I. J. Catecholamine Metabolism: Basic Aspects and Clinical Significance. Pharmacol. Rev., Baltimore, v. 37, n. 4, p. 333-364, 1985.

LUNDBERG, U. Catecholamines. In: FINK, G., Ed., Encyclopedia of Stress. New York: Academic Press, 2000. v. 1, p. 408-413.

MANO, K., AKBARZADEH, A., TOWNLEY, R. G. Effect of hidrocortisone on beta-adrenergic receptors in lung membranes. Life Sci., Oxford, v. 25, n. 22, p. 1925-1930, 1979.

MARCONDES, F. K. Influência do ciclo estral sobre as respostas hormonais de ratas submetidas a estresse. Campinas, 1998. 62p. [Tese de Doutorado - Instituto de Biologia - Universidade Estadual de Campinas].

MARCONDES, F. K. Influência do sexo e das fases do ciclo estral sobre a reação de estresse em ratos. Campinas, 1995. 58p. [Dissertação de Mestrado - Instituto de Biologia - Universidade Estadual de Campinas].

MARCONDES, F. K., BIANCHI, F. J., TANNO, A. P. Determination of the Estrous Cycle Phases of Rats: Some Helpful Considerations. Braz. J. Biol., no prelo, 2002.

MARCONDES, F. K, VANDERLEI, L. C. M., LANZA, L. L. B., SPADARI-BRATFISCH, R. C. Stress-induced subsensitivity to catecholamines depends on the estrous cycle. Can. J. Physiol. Pharmacol., Ottawa, v. 74, n. 6, p. 663-669, 1996.

McCANN, S. M., ANTUNES-RODRIGUES, J., FRANCI, C. R., ANSELMO-FRANCI, J. A., KARANTH, S. , RETTORI, V. Role of the hypothalamic pituitary adrenal axis in the control of the response to stress and infection. Braz. J. Med. Biol. Res., São Paulo, v. 33, n. 10, p. 11211131, 2000.

McEWEN, B. S. Definitions and Concepts of Stress. In: FINK, G., ed. Encyclopedia of Stress. New York: Academic Press, 2000. v. 3, p. 408-509.

MOURA, M. J. C. S , MARCONDES, F. K. Influence of estradiol and progesterone on the sensitivity of rat thoracic aorta to noradrenaline. Life Sci., Oxford, v. 68, n. 8, p. 881-888, 2001.
ÖSTMAN-SMITH, I. Adaptive changes in the sympathetic nervous system and some effector organs of the rat following long term exercise or cold acclimation and the role of cardiac sympathetic nerves in the genesis of compensatory cardiac hipertrophy. Acta Physiol. Scand., Oxford, Supplement 477, p. 1-118, 1979.

RODRIGUES, M. L. V., MARCONDES, F. K. , SPADARIBRATFISCH, R. C. Relationship between sensitivity to adrenaline, plasma corticosterone level and estrous cycle in rats. Can. J. Physiol. Pharmacol., Ottawa, v. 73, n. 5, p. 602-607, 1995.

SALT, P. J. Inhibition of noradrenaline uptake ${ }_{2}$ in the isolated rat heart by steroids, clonidine and methoxylated phenylethylamines. Eur. J. Pharmacol., Amsterdam, v. 20, n. 3, p. 329-340, 1972.

SHORS, T. J., PICKETT, J., WOOD, G. , PACYNSKI, M. Acute Stress Persistently Enhances Estrogen Levels in the Female Rat. Stress., Malaysia, v. 3, n. 2, p. 163-171, 1999.

SMITH, M. S., FREEMAN, M. E. , NEILL, J. D. The control of progesterone secretion during the estrous cycle and early pseudopregnancy in the rat: prolactin, gonadotrofin and steroids levels associated with rescue of the corpus luteum of pseudopregnancy. Endocrinology., Baltimore, v. 96, n. 1, p. 219-226, 1975.

SPADARI, R. C., BASSANI, R. A., DE MORAES, S. Supersensitivity to isoprenaline and epinephrine in right atria isolated from rats submitted to a single swimming session. Gen. Pharmac., Oxford, v. 19, n. 1, p. 129-135, 1988.

SPADARI, R. C. , DE MORAES, S. Repeated swimming stress and responsiveness of isolated rat pacemaker to chronotropic effect of noradrenaline and isoprenaline: role of adrenal corticosteroids. Gen. Pharmacol., Oxford, v. 19, n. 4 , p. $553-557,1988$.

SPADARI-BRATFISCH, R. C., NUNES, I. S., VANDERLEI, L. C. M, MARCONDES, F K. Evidence for $b_{2}$-adrenoceptors in right atria from female rats submitted to footshock stress. Can J. Physiol. Pharmacol., Ottawa, v. 77, n. 6, p. 432-440, 1999. 
VAN ROSSUM, J. M. Cumulative dose-response curves. II. Technique for the making $f$ dose-response curves in isolated organs and the evaluation of the drug parameters. Arch. Int. Pharmacodyn. Ther., Ghent, v. 143, p. 229-230, 1963.

VANDERLEI, L. C. M, MARCONDES, F. K., LANZA, L. L. B. , SPADARI-BRATFISCH, R. C. Influence of the estrous cycle on the sensitivity to catecholamines in right atria from rats submitted to footshock stress. Can. J. Physiol Pharmacol., Ottawa, v. 74, n. 6, p. 670-678, 1996.
ZANESCO, A. SPADARI-BRATFISCH, R. C., BARKER, L. A. Sino-aortic denervation causes right atrial beta adrenoceptor down-regulation. J. Pharmacol. Exp. Ther., London, v. 280, n. 2, p. 677-685, 1997.

Recebido para publicação em 15 de outubro de 2001. 